\title{
Avaliação de modelos digitais de elevação para aplicação em um mapeamento digital de solos
}

\author{
César S. Chagas ${ }^{1}$, Elpídio I. Fernandes Filho ${ }^{2}$, Márcio F. Rocha ${ }^{3}$, Waldir de Carvalho Júnior ${ }^{4}$ \& Nestor C. Souza Neto ${ }^{4}$
}

\begin{abstract}
RESUMO
No Brasil, normalmente os modelos digitais de elevação (MDEs) são produzidos pelos próprios usuários e pouca atenção tem sido dada às suas limitações, como fonte de informação espacial. Este estudo propôs avaliar diferentes MDEs para subsidiar a escolha do modelo apropriado para derivar atributos topográficos utilizados em um mapeamento digital de solos, por redes neurais artificiais. A avaliação constou da determinação da raiz quadrada do erro médio quadrático da elevação (RMSE); análise das depressões espúrias; comparação entre drenagem mapeada e drenagem numérica, curvas de nível derivadas e curvas de nível originais, e análise das bacias de contribuição derivadas. Os resultados obtidos demonstraram que apenas o RMSE não foi suficiente para avaliar a qualidade desses modelos. O MDE, derivado de curvas de nível (CARTA, obtido com a utilização do módulo TOPOGRID) apresentou qualidade superior aos MDEs derivados de sensores remotos (ASTER e SRTM). A análise qualitativa também identificou que o MDE CARTA é superior aos demais, pois estes apresentaram grande quantidade de erros que podem comprometer o estabelecimento das relações entre atributos do terreno e as condições locais de solos.
\end{abstract}

Palavras-chave: TOPOGRID, ASTER, SRTM

\section{Evaluation of digital elevation models for application in a digital soil mapping}

\begin{abstract}
In Brazil, the digital elevation models (DEMs) are usually produced by users themselves and little attention has been given to their limitations as source of spatial information. The objective of this study was to evaluate different DEMs to help in choosing an appropriate model to derive topographical attributes used in a digital soil mapping based on a neural networks approach. The evaluation consisted of the following analysis: determination of root mean square error (RMSE) of elevation; analysis of the spurious depressions; comparison between mapped drainage and numeric drainage and between derived contour lines and original contour lines; and analysis of the derived contribution basins. The results demonstrated that RMSE was not enough to evaluate the quality of these models. DEMs derived from contour lines (CARTA, obtained using the TOPOGRID module) presented better quality than those derived from remote sensors (ASTER and SRTM). These presented great amount of errors that can negatively affect the establishment of relationships between topographical attributes and local conditions of soils.
\end{abstract}

Key words: TOPOGRID, ASTER, SRTM

\footnotetext{
1 Embrapa Solos. Rua Jardim Botânico, 1024, Jardim Botânico, CEP 22460-000, Rio de Janeiro, RJ. Fone: (21) 2179-4546. E-mail: chagas.rj@gmail.com 2 DPS/UFV, CEP 36570-000, Viçosa, MG. Fone: (31) 3899-1040. E-mail: elpidio@ufv.br

3 IF/UFRRJ, CEP 23890-000, Seropédica, RJ. Fone: (21) 2682-1128. E-mail: marciorocha@ufrrj.br

${ }_{4}^{4}$ Embrapa Solos. CEP 22460-000, Rio de Janeiro, RJ. Fone: (21) 2179-4582. E-mail: waldircj@cnps.embrapa.br; (21) 2179-4616, nestor@cnps.embrapa.br
} 


\section{INTRODUÇÃO}

A demanda por modelos digitais de elevação (MDEs) para subsidiar estudos em várias áreas da pesquisa ambiental, tem aumentado significativamente; no entanto, diferentemente de alguns países desenvolvidos não existe, no Brasil, um órgão responsável pela elaboração e normatização dos procedimentos utilizados na criação desses modelos os quais, normalmente, são produzidos pelos próprios usuários e pouca atenção é dada às suas limitações como fonte de informação espacial.

A fonte de dados mais comum para a geração de MDEs no Brasil ainda são as curvas de nível e, de forma complementar, a rede hidrográfica, obtidas em cartas topográficas, principalmente aquelas elaboradas pelo Instituto Brasileiro de Geografia e Estatística - IBGE; entretanto, as escalas normalmente disponíveis não são adequadas para estudos mais detalhados; por esta razão, dados oriundos de imagens de sensores remotos estão sendo cada vez mais utilizados para a geração desses modelos.

Os MDEs, gerados a partir de pares de imagens estereoscópicas de sensores óticos orbitais, apresentam algumas vantagens significativas sobre outras fontes de dados, como: a disponibilidade de dados em nível mundial; grande cobertura de área por cena; resolução moderadamente elevada; processamento mais rápido através de softwares sofisticados e pouco esforço manual; baixo custo de processamento e capacidade de aquisição em qualquer condição ambiental, no caso de sensores ativos (Subramanian et al., 2005). Apesar das vantagens apresentadas, os métodos utilizados medem as elevações com erros significativos, os quais dependem da limitação inerente dos instrumentos de observação e da rugosidade e declividade da superfície; também carecem de pontos de controle localizados precisamente, para minimizar o erro sistemático, o que nem sempre é fácil de ser obtido, especialmente em regiões remotas (Dixon, 1995).

A utilidade e a validade dos resultados derivados dos MDEs estão estreitamente relacionadas com a qualidade do modelo original. É muito freqüente, na bibliografia, a ausência de informações sobre as características desses modelos utilizados nas aplicações, em especial quando eles são construídos pelos próprios investigadores e não provêm de fontes oficiais de organismos qualificados (Felicísimo, 1994).

A qualidade de um MDE depende do tipo e da magnitude dos erros a ele relacionados. É importante considerar que os erros são inevitáveis, visto que os MDEs são visões simplificadas da realidade, submetidas a um processo de generalização. Os parâmetros modelados são, portanto, inerentemente imprecisos embora sua representação possa realizar-se com mais ou menos exatidão, dependendo do método de coleta de dados utilizados; além disso, os modelos são criados, distribuídos e usados muito freqüentemente sem qualquer referência à magnitude do erro ou ao método aplicado para sua detecção ou correção (Felicísimo, 1994).

O método tradicional de avaliação da qualidade de um MDE é confrontar uma amostra de pontos de elevação do MDE produzido contra pontos de elevação conhecidos, provenientes de uma fonte de dados mais acurada. O grau de concordância entre esses MDEs é então referido como raiz quadrada do erro médio quadrático (RMSE) da elevação.

Para Wise (2000), dois problemas estão relacionados a esta abordagem, em que o primeiro se refere ao fato de que, normalmente, esta é baseada em poucos pontos de amostras (20 a 30 pontos) e o outro, talvez o mais importante, é que esta abordagem não auxilia na identificação da fonte do erro; apesar disso, esta abordagem tem sido muito utilizada na comparação da qualidade de MDEs produzidos de diferentes fontes e métodos de interpolação.

Erros presentes em um MDE podem confundir as relações esperadas entre atributos do terreno computados e as condições locais. Esses problemas podem ser amplificados quando atributos de primeira e segunda ordens são computados (Bolstad \& Stowe, 1994) sendo encontrados, usualmente, problemas mais sérios quando atributos secundários são derivados.

O presente estudo teve como objetivo avaliar MDEs gerados a partir de dados do sensor VNIR do ASTER (Advanced Spaceborne Thermal Emission and Reflection Radiometer), do SRTM (Shuttle Radar Topography Mission) e de cartas topográficas do IBGE na escala de 1:50.000, em uma região montanhosa no Noroeste do Estado do Rio de Janeiro. Tal avaliação tem por finalidade auxiliar na escolha do modelo digital de elevação mais adequado para derivar atributos topográficos primários e secundários para utilização em um mapeamento digital de solos por redes neurais.

\section{MATERIAL E MÉTODOS}

O estudo foi desenvolvido na bacia hidrográfica do Rio São Domingos, afluente do rio Muriaé, ambos pertencentes ao complexo hidrográfico do Rio Paraíba do Sul. A bacia, que apresenta uma área aproximada de 28.000 ha, está situada entre as coordenadas UTM 7.620.700 e 7.647.200 mN e 183.000 e $210.800 \mathrm{mE}$, zona 24S, englobando a totalidade do município de São José de Ubá e parte do município de Itaperuna, no Noroeste do Estado do Rio de Janeiro (Figura 1). A área da bacia do Rio São Domingos é constituída de áreas planas com elevações em torno de $100 \mathrm{~m}$ e áreas extremamente íngremes, que alcançam quase $1000 \mathrm{~m}$ de altitude. Embora apresente grande variação altimétrica, cerca de $84 \%$ da área da bacia, apresenta altitudes que variam entre 80 e 300 m, com elevação média de 217 m.

\section{Modelos digitais de elevação}

Foram utilizados, neste estudo, um MDE gerado a partir de curvas de nível e dois derivados a partir de métodos baseados em sensoriamento remoto orbital, os quais foram avaliados com relação à qualidade vertical que apresentam e ao potencial para se derivarem atributos do terreno que reflitam os principais processos geomórficos, climáticos e hidrológicos que ocorrem na área estudada. Para possibilitar a comparação, todos os MDEs foram gerados com uma resolução espacial de $30 \mathrm{~m}$.

O MDE obtido a partir de curvas de nível foi elaborado utilizando-se os dados relativos às curvas de nível, com 


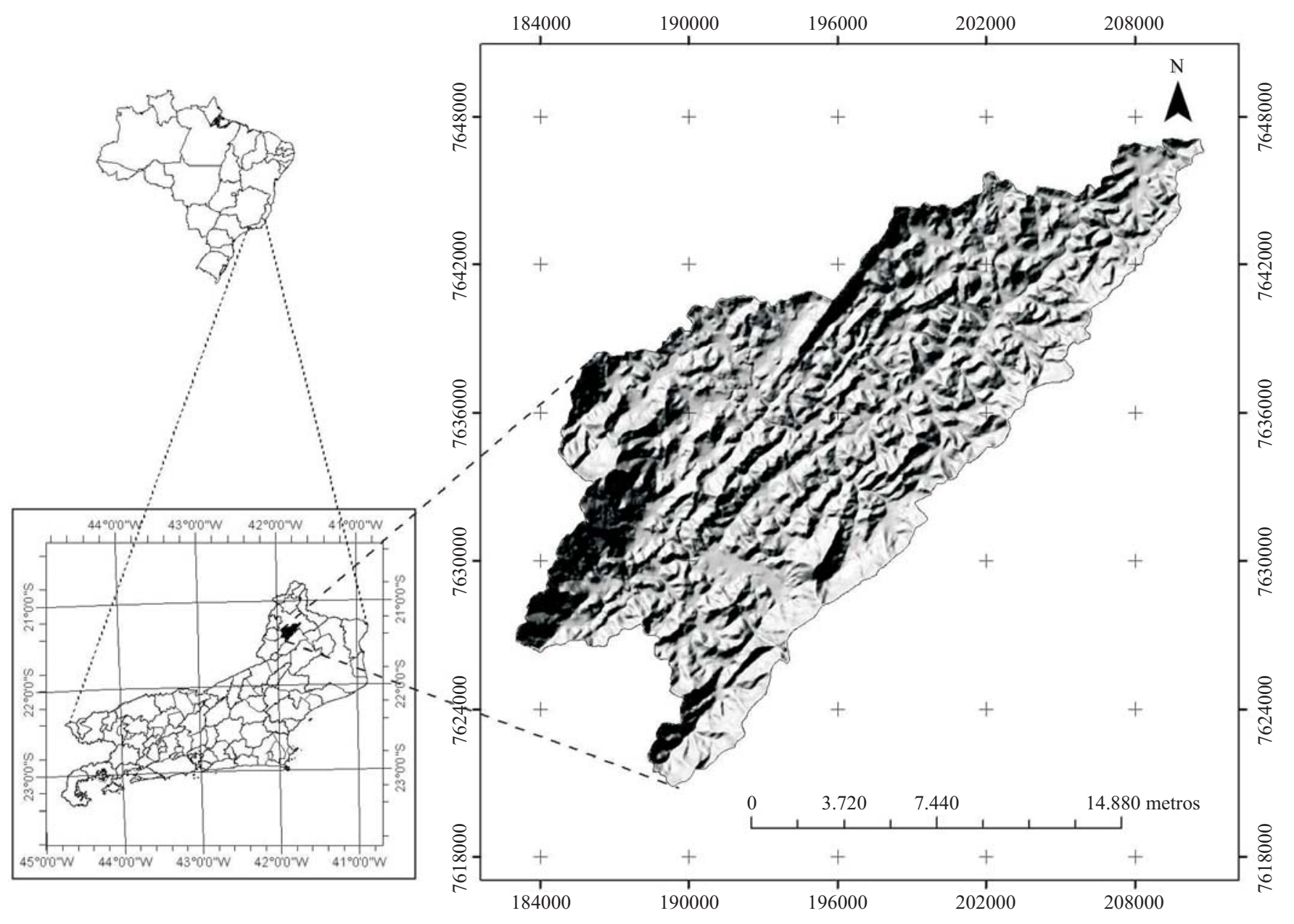

Figura 1. Localização da bacia do Rio São Domingos no Estado do Rio de Janeiro, destacando-se a rugosidade de seu relevo

eqüidistância vertical de $20 \mathrm{~m}$, hidrografia e pontos cotados contidos nas cartas topográficas do IBGE, na escala de 1:50.000, no formato digital, referentes às Folhas Miracema (SF-23-X-D-III-4) e São João do Paraíso (SF-24-G-I3) previamente unidas, dando origem aos planos de informação hidrografia, curvas de nível e pontos cotados.

Existem numerosos métodos disponíveis para produção de MDEs a partir de curvas de nível, os quais podem ser distinguidos pela maneira como tentam modelar a superfície, usando a informação das curvas (Wise, 2000). No presente estudo se utilizou o método baseado no ajustamento da superfície, através do módulo TOPOGRID do software ARC/ INFO. As curvas de nível e pontos cotados, juntamente com a hidrografia, devidamente editada para assegurar que todos os rios estavam fluindo para a foz, mais o limite da área, foram utilizados como dados de entrada na função GRID do ARC/INFO para geração do MDE CARTA.

Os MDEs obtidos a partir de sensores remotos utilizados, foram o MDE do SRTM e o MDE do ASTER. O MDE do SRTM foi disponibilizado gratuitamente a partir do servidor de ftp do USGS (ftp://edcsgs9.cr.usgs.gov/pub/data/srtm/). Para compor a área de estudo obtiveram-se duas cenas do SRTM (S22W042 e S22W043) e, em seguida, se criou um mosaico a partir dessas, de onde se recortou a área de interesse desse estudo. Finalmente, a área foi analisada para a verificação da existência de valores negativos e/ou áreas sem valores de altitudes. Durante o processamento final o MDE SRTM_90 foi, então, projetado no ArcGIS Desktop 9.0 para a projeção Universal Transversa de Mercator (UTM), Zona 24S e datum Córrego Alegre.

Para se adequar aos objetivos do trabalho e possibilitar a comparação com os demais MDEs da área e se considerando que a base de dados SRTM foi originalmente produzida com a resolução de 30 m, o MDE SRTM (90 m) foi reamostrado para $30 \mathrm{~m}$, usando-se o ArcGIS Desktop 9.0, para fornecer um MDE com resolução compatível com a das cartas do IBGE (MDE SRTM_30). Na reamostragem preferiu-se realizar uma interpolação bilinear para determinar novos valores para as células do grid, com base na média obtida para os quatro pixels vizinhos mais próximos ao pixel de saída, semelhante ao procedimento utilizado por Crepani \& Medeiros (2004).

Para a geração do MDE ASTER se utilizou uma imagem deste sensor, de fevereiro de 2002, correspondente ao nível $1 \mathrm{~B}$, com $7 \%$ de cobertura de nuvens. A partir desta imagem se fez um recorte da área de interesse para o estudo e a correção geométrica com base nas cartas topográficas do IBGE, para o sistema de coordenadas UTM (Universal Transversa de Mercator), datum Córrego Alegre e zona 24S.

O MDE ASTER foi gerado utilizando-se o módulo 
AsterDTM 2.2 do software ENVI 4.2. O MDE ASTER gerado neste estudo, é um MDE relativo já que não se dispunha de pontos de controle terrestres de elevada exatidão para criação de um MDE absoluto.

\section{Avaliação da Qualidade dos MDEs}

Os MDEs foram avaliados quantitativa e qualitativamente. Para determinar o nível de erro ou incerteza contido nos conjuntos de dados avaliados, utilizaram-se 18 pontos de elevação (pontos de controle) obtidos diretamente no campo pela DRM-RJ com um GPS modelo Geoexplorer ${ }^{\circledR} 3$ da marca TRIMBLE e 194 pontos com um GPS modelo ProMark II da marca ASHTECH. Os dados obtidos por esses GPS foram posteriormente processados com a finalidade de se atingir uma precisão submétrica.

A medida da qualidade de MDEs é usualmente apresentada na forma da raiz quadrada do erro médio quadrático (RMSE) da elevação, obtido conforme a Eq. 1.

$$
\operatorname{RMSE}=\sqrt{\frac{\sum_{\mathrm{i}=1}^{\mathrm{n}} \mathrm{d}_{\mathrm{i}}^{2}}{\mathrm{n}}}
$$

donde d é a diferença de elevação entre os MDEs avaliados e os pontos de controle e n é o número de pontos de elevação testados. Segundo Holmes et al. (2000), o RMSE é medido a partir de pontos de amostra discretos e é comumente utilizado para estimar o erro ou a incerteza nos locais em que o erro não foi medido diretamente. Para um MDE, o RMSE se refere ao grau de diferença entre os valores interpolados e os valores de elevação mais prováveis, de modo que o termo elevação verdadeira não deve ser utilizado já que, normalmente, este é considerado desconhecido.

Comparações entre os MDEs ASTER e SRTM com o MDE CARTA, foram realizadas por meio da análise dos perfis de elevação obtidos com a opção Raster/Profile Tools/ Spatial Profile do ERDAS Imagine 8.5.

Visto que a maioria das aplicações dos MDEs depende de representações da forma da superfície e estrutura da drenagem, medidas absolutas dos erros de elevação não fornecem uma avaliação completa da qualidade de um MDE (Wise, 2000). Um grande número de técnicas descritivas para avaliação da qualidade de dados tem sido desenvolvido; essas técnicas são medidas não clássicas da qualidade de dados que oferecem meios para análise sem a utilização de pontos de referência de elevada exatidão (Hutchinson \& Gallant, 2000).

Em muitas situações o desenvolvimento do solo ocorre em resposta ao modo como a água se move, através e sobre a paisagem. Os atributos do terreno podem caracterizar os caminhos do fluxo e as interações com os atributos do solo; assim, para contornar as limitações da utilização do RMSE na avaliação dos MDEs realizou-se uma avaliação da sua qualidade, em termos da representação dos aspectos da superfície, conforme sugerido por Hutchinson \& Gallant (2000) e Wise (2000), que são: a) análise do número e extensão das depressões espúrias (células cercadas por outras com valores de elevação maiores); b) comparação entre a drenagem mapeada e a drenagem derivada pelos diferentes MDEs; c) comparação entre as curvas de nível derivadas e as curvas de nível originais e d) análise das bacias de contribuição derivadas.

As depressões espúrias nos MDEs avaliados, foram detectadas em número e extensão utilizando-se o módulo Spatial Analyst do ArcGIS Desktop 9.0. A rede de drenagem foi derivada para cada MDE, após a remoção das depressões espúrias, usando-se as funções FlowDirection e FlowAccumulation no módulo Hydrology, que faz parte da ferramenta Spatial Analyst do ArcGIS Desktop 9.0. O valor de escoamento acumulado necessário para gerar a rede de drenagem numérica com aproximadamente o mesmo nível de detalhe da rede de drenagem mapeada, foi obtido por meio de tentativa e erro.

De acordo com Hutchinson \& Gallant (2000), as curvas de nível derivadas de um MDE fornecem uma avaliação sensitiva sobre a estrutura do terreno, o que é bastante útil, devido a sua sensibilidade aos erros de elevação na fonte de dados. Deste modo se realizou uma comparação visual entre as curvas de nível derivadas a partir dos MDEs com as curvas de nível originais para detectar a presença de artefatos nos primeiros. Neste procedimento também se lançou mão do módulo Spatial Analyst do ArcGIS Desktop 9.0. Deficiências na qualidade de um MDE podem ser detectadas pela derivação das bacias de contribuição (Wise, 2000); ressaltase que elas foram obtidas através da função Watershed, no módulo Hydrology do ArcGIS Desktop 9.0.

\section{RESULTADOS E DISCUSSÃO}

Os resultados da Tabela 1 mostram grande semelhança entre os valores de RMSE produzidos pelo MDE CARTA e pelo MDE SRTM os quais, por outro lado, são bastante distintos daqueles obtidos para o MDE ASTER, com os primeiros apresentando valores consideravelmente melhores.

Tabela 1. Resultados estatísticos para os diferentes MDEs estudados

\begin{tabular}{cccccc}
\hline MDE & $\begin{array}{c}\text { Valor } \\
\text { Mínimo }\end{array}$ & $\begin{array}{c}\text { Valor } \\
\text { Máximo }\end{array}$ & Média & $\begin{array}{c}\text { Desvio } \\
\text { padrão }\end{array}$ & $\begin{array}{c}\text { RMSE(a) } \\
(\mathbf{m})\end{array}$ \\
CARTA & 69 & 913 & 212 & 121 & 14 \\
ASTER & 102 & 877 & 236 & 114 & 37 \\
SRTM & 82 & 901 & 219 & 119 & 12 \\
\hline
\end{tabular}

(a) Obtido pela diferença com os pontos de elevação de referência

O MDE ASTER produziu um valor de RMSE bastante elevado, na comparação com os pontos de elevação de referência (37 m) (Tabela 1), apresentando o pior desempenho entre os MDEs avaliados. Esses resultados são bastante discordantes daqueles obtidos em outros estudos, em que se obtiveram resultados mais satisfatórios (Hirano et al., 2003; Cuartero et al., 2004; Poli et al., 2004). Prováveis explicações para esses resultados incluem as características do terreno, a presença de nuvens nas imagens, a falta de pontos de controle terrestres adequados e até mesmo o próprio software empregado para obtenção do MDE; entretanto, Kääb 
et al. (2002), obtiveram um RMSE de aproximadamente $60 \mathrm{~m}$ para uma área montanhosa na Nova Zelândia, com o erro máximo alcançando até $500 \mathrm{~m}$. Análises realizadas mostraram grandes erros no MDE derivado do ASTER nas encostas íngremes. De acordo com os autores, esses erros são previsíveis considerando-se o fato de que tais encostas ficam totalmente escondidas ou sombreadas quando da passagem do sensor que obtém a banda 3B, ou pelo menos são grandemente distorcidas, condições em que as encostas recebem menos iluminação solar reduzindo, assim, o contraste da imagem e aumentando a probabilidade de falhas na correlação entre as bandas 3N e 3B.

$\mathrm{Na}$ área estudada se encontram encostas extremamente íngremes, que podem atingir mais de $900 \mathrm{~m}$, adjacentes a áreas planas com altitudes em torno de $100 \mathrm{~m}$; assim, os problemas descritos por Kääb et al. (2002), podem ter interferido negativamente para o elevado valor do RMSE do MDE ASTER.

Erros grosseiros em MDEs gerados a partir de dados de sensoriamento remoto, podem ser atribuídos a muitos fatores, como: ruídos nos processos de aquisição e transferência dos dados, erros na geometria da órbita ou, ainda, a presença de nuvens nas imagens; deste modo, a presença de $7 \%$ de nuvens nas imagens ASTER utilizadas neste estudo pode ter contribuído para reduzir a qualidade final do MDE.

Avaliações da qualidade vertical de MDEs derivados do sensor ASTER a partir de estereocorrelação, indicam que valores de RMSE entre \pm 7 e \pm 15 m podem ser alcançados quando se utilizam softwares adequados, imagens de boa qualidade e pontos de controle terrestres adequados (Hirano et al., 2003). No presente estudo a falta de pontos de controle adequados para geração do MDE ASTER foi o fator que mais contribuiu para a baixa qualidade do modelo.

Alguns estudos mostram que o software utilizado para geração do MDE ASTER exerce influência marcante sobre os valores de RMSE obtidos. Cuartero et al. (2004), obtiveram resultados distintos para valores de RMSE utilizando dois diferentes softwares na geração de MDEs ASTER. O software Ortho Engine (Geomatica), que tem um módulo específico para dados do ASTER apresentou, para uma mesma área, resultados melhores (RMSE de 12,6 m) que o módulo Ortho Base do ERDAS IMAGINE (RMSE de 34,8 m); assim, o software utilizado na geração do MDE ASTER deste estudo (AsterDTM 2.2 do software ENVI 4.2) também pode ter influenciado nos resultados obtidos.

Druzina et al. (2005) obtiveram resultados semelhantes ao comparar um MDE derivado do ASTER com pontos de controle terrestre e um MDE derivado de curvas de nível em uma região montanhosa no sul de Santa Catarina e nordeste do Rio Grande do Sul. Nas duas comparações, o MDE ASTER representou uma superfície mais elevada que os pontos de controle terrestre e que a do MDE derivado de curvas de nível.

Resultados discordantes obtiveram Kamp et al. (2003); Bolch \& Kamp (2003) e Eckert \& Kellenberger (2002) que, em geral, encontraram valores de elevação mais baixos que os pontos de controle utilizados como referência. Kamp et al. (2003), atribuíram esses resultados à falta de pontos de controle para geração do MDE e ao procedimento de suavização do software empregado (Geomatica).

Os resultados da Tabela 1 mostram que o MDE SRTM produziu um valor de RMSE bastante satisfatório, na comparação com os pontos de elevação de referência (12 m), sendo próximo ao resultado obtido pelo MDE CARTA. Ressalta-se que, para esta avaliação, os dados originais do SRTM foram reamostrados para $30 \mathrm{~m}$, de modo a permitir uma comparação com os demais MDEs; apesar disto, os resultados obtidos foram superiores aos demais.

Segundo USGS (2005), a exatidão vertical esperada para os dados do SRTM é de $\pm 16 \mathrm{~m}$; portanto, os resultados alcançados estão de acordo com o especificado. Barros et al. (2005), obtiveram um RMSE de 23,94 m para um MDE do SRTM em uma área litorânea montanhosa no sul do Estado do Rio de Janeiro, com resíduo máximo de 48,3 m em relação aos pontos de controle utilizados, porém alguns trabalhos têm apontado para a possibilidade de obtenção de precisões melhores que as inicialmente estimadas (Koch et al., 2002; Santos et al., 2005). Santos et al. (2005) compararam, em estudo realizado na região amazônica, dados do SRTM com MDEs gerados a partir de cartas topográficas, em diferentes escalas, utilizando um conjunto de pontos de controle. Em todas as situações avaliadas o MDE do SRTM apresentou melhores resultados altimétricos em relação ao MDE originado de cartas topográficas, com valores do erro médio quadrático de 10,74 m, para a escala de 1:50.000, quando se considerou a totalidade dos pontos de controle disponíveis.

Os resultados obtidos para o MDE SRTM podem ser influenciados pelas características do terreno. Resultados de estudos realizados por Kocak et al. (2004) e Gerstenecker et al. (2005), mostraram que a exatidão de MDEs derivados de sensores remotos é muito dependente da inclinação do terreno, sendo a qualidade mais baixa em áreas declivosas que em áreas planas, razão por que Falorni et al. (2005) sugerem que, em terrenos muito elevados e íngremes, a exatidão especificada de $16 \mathrm{~m}$ para MDEs do SRTM deva ser considerada apenas como diretriz.

Na Figura 2 estão os perfis de elevação dos MDEs avaliados, na qual é possível verificar, também, a grande diferença nos valores de elevação entre o MDE ASTER e o MDE CARTA, sendo esta diferença, em grande parte, positiva, ou seja, o MDE ASTER representa uma superfície mais elevada que o MDE CARTA. A média e o desvio padrão obtidos para esta diferença foram de 24 e $28 \mathrm{~m}$, respectivamente.

Por sua vez, as maiores discordâncias entre o MDE SRTM e o MDE CARTA nas seções longitudinais ocorrem nas partes mais baixas e planas da área, mas as maiores diferenças nos valores de elevação entre eles se dão exatamente nas áreas com as maiores declividades.

Jarvis et al. (2004), observaram tendência semelhante nos dados de elevação influenciada pela orientação da encosta, ao avaliarem um MDE do SRTM para Honduras porém, neste estudo, as diferenças positivas em relação ao MDE oriundo de cartas topográficas, estavam presentes nas encostas norte, nordeste ou leste, enquanto as negativas foram observadas nas encostas sul, sudeste ou oeste; esses resultados, segundo os autores, foram atribuídos ao efeito do ângulo de 


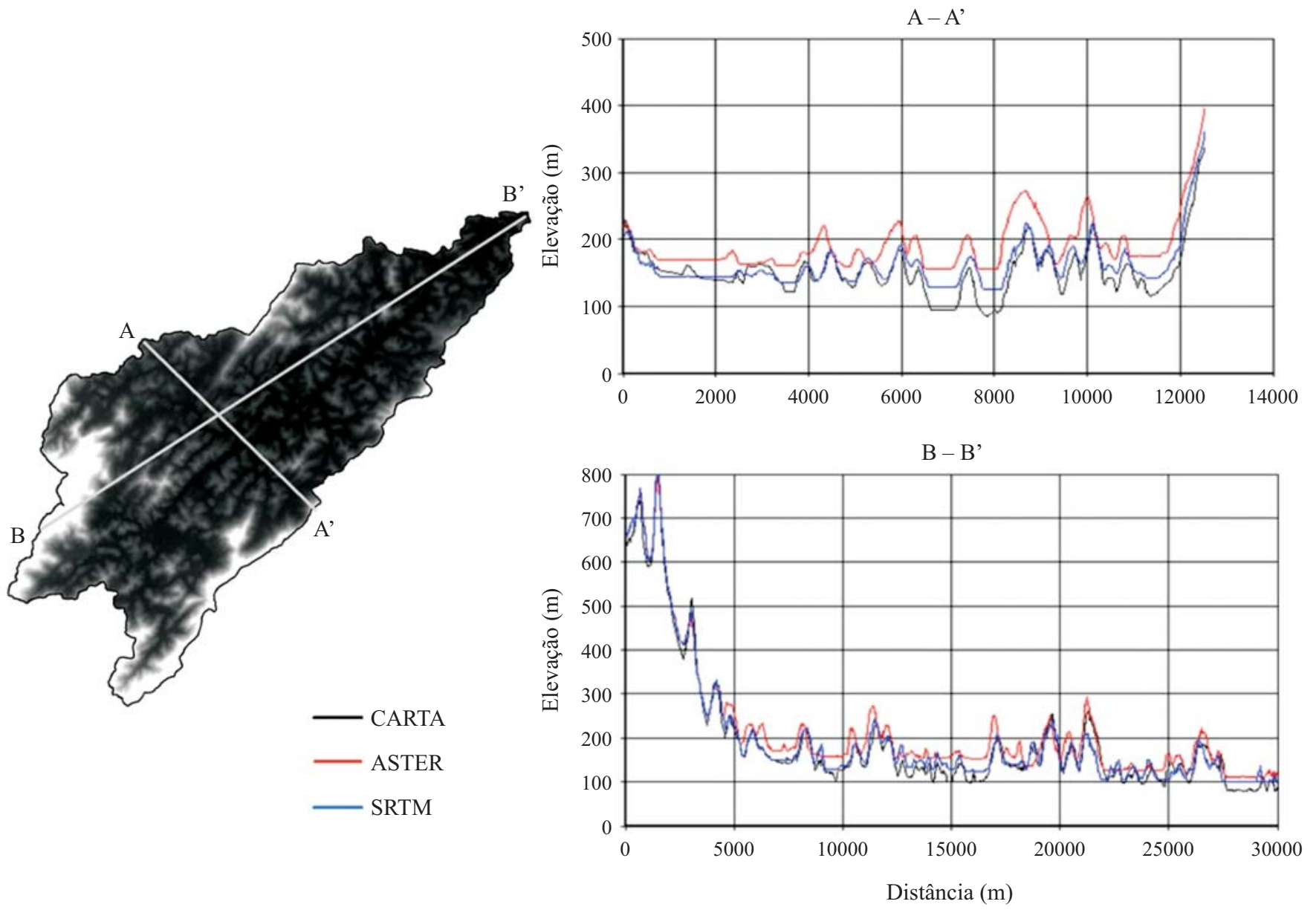

Figura 2. Perfis de elevação dos MDEs estudados

incidência das imagens de radar originais, usadas para produzir o MDE do SRTM.

A derivação da rede de drenagem é sensivelmente afetada pela presença de depressões espúrias em um MDE, que podem comprometer análises futuras (Wise, 2000). Assim, a avaliação qualitativa constou, inicialmente, da determinação do número e extensão das depressões em cada MDE.

A Tabela 2 mostra haver uma diferença marcante no número e na extensão das depressões espúrias entre o MDE derivado das curvas de nível e os derivados dos sensores remotos. Quando se considera o número de depressões, verifica-se que os MDEs derivados de sensores remotos apresentam aproximadamente 5 vezes mais que o observado no MDE CARTA. Com relação ao número de pixels afetados pelas depressões, as diferenças são mais marcantes, cerca de 8 vezes, quando se compara o MDE SRTM com o MDE CARTA.

Considerando-se apenas o número e a extensão das de-

Tabela 2. Número e extensão das depressões espúrias dos MDEs avaliados

\begin{tabular}{cccc}
\hline MDE & $\begin{array}{c}\text { Número de } \\
\text { depressões }\end{array}$ & $\begin{array}{c}\text { Número de pixels } \\
\text { nas depressões }\end{array}$ & $\begin{array}{c}\text { \% em relação } \\
\text { ao grid total }\end{array}$ \\
CARTA & 394 & 781 & 0,10 \\
ASTER & 2104 & 4308 & 0,53 \\
SRTM & 2057 & 6601 & 0,81 \\
\hline
\end{tabular}

pressões espúrias, tem-se que o melhor MDE produzido é o MDE CARTA com apenas 394 depressões, que englobam 781 pixels. Embora o MDE CARTA tenha sido produzido com a utilização do módulo TOPOGRID, o número de depressões espúrias nele presente é relativamente alto; uma possível explicação para esses resultados, levantada por Wise (2000), é a natureza do terreno, que combina encostas muito íngremes com vales planos, o que leva à formação de depressões, principalmente nos limites entre os vales e as encostas (Figura 3).

Os MDEs ASTER e SRTM apresentaram um número elevado de depressões espúrias, disseminadas por toda a área (Figura 3B e 3C). Apesar de serem numerosas, o número de pixels envolvidos em cada depressão é reduzido, normalmente de 1 a 2 pixels. Somente em poucos casos, principalmente no MDE SRTM, o número de pixels é superior a 2, como pode ser observado no canto superior direito da Figura 3C. O elevado número de depressões espúrias nesses MDEs, muito comum em dados derivados de sensores remotos, pode ser atribuído a erros grosseiros oriundos do método de coleta de dados utilizado por esses sensores (Hengl et al., 2004).

A rede de drenagem derivada a partir de um MDE representa o padrão de fluxo acumulado elevado e a localização potencial da malha dos rios. As redes de drenagem foram derivadas a partir dos MDEs sem depressões espúrias e comparadas visualmente com a drenagem mapeada (1:50.000). 
A.

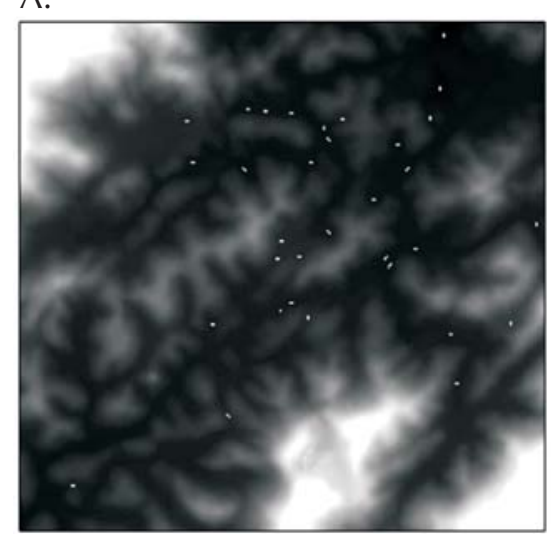

B.

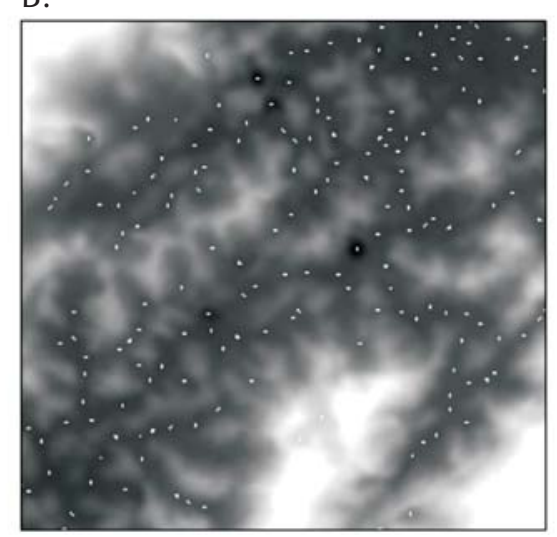

C.

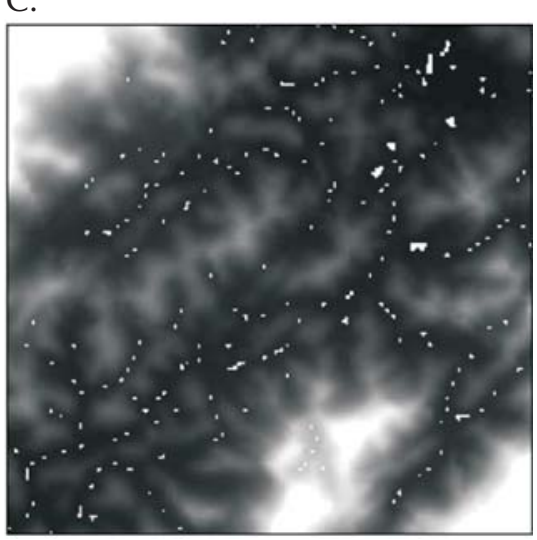

Figura 3. Localização das depressões espúrias em uma parte dos MDEs avaliados. A - MDE CARTA; B - MDE ASTER e C - MDE SRTM

O valor de escoamento acumulado necessário para gerar as redes de drenagem com aproximadamente o mesmo nível de detalhe da drenagem mapeada, foi estimado em 500. Os resultados são mostrados na Figura 4.

A melhor concordância entre a rede de drenagem derivada e a drenagem mapeada, foi obtida pelo MDE CARTA, corroborando com as afirmativas de que os modelos gerados pelo interpolador TOPOGRID são caracterizados por apresentarem boa coincidência entre a sua drenagem derivada e a mapeada (Ribeiro, 2003); contudo, diferenças no curso exato para alguns canais podem ser notadas. De maneira geral, não se constata, na drenagem derivada deste MDE, a presença expressiva de erros grosseiros, tal como o cruzamento com as curvas de nível.

As redes de drenagem derivadas dos MDEs ASTER e SRTM mostram baixa concordância com a drenagem mapeada evidenciando, nesses casos, a presença marcante de erros na fonte de dados utilizada, nas quais facilmente se observam drenagens que cruzam curvas de nível, e outras paralelas, que indicam a presença de artefatos nesses MDEs (Figura 4), principalmente no MDE ASTER e um pouco menos no MDE SRTM.

Os resultados da comparação visual entre as curvas de nível geradas a partir dos MDEs com as curvas de nível ori- ginais, são mostrados na Figura 5.

O objetivo que se busca na criação de um MDE é a obtenção de um modelo que contenha, o máximo possível, detalhes topográficos, preservando sempre as características do terreno; assim, as curvas de nível derivadas generalizaram os dados em quantidades variadas. A melhor concordância com as curvas de nível digitalizadas foi obtida pelo MDE CARTA, conforme observado na Figura 5. Os picos foram mantidos e somente pequenas variações são notadas com relação às linhas originais, podendo o MDE CARTA ser considerado, portanto, de qualidade superior aos demais, para este critério.

Os MDEs ASTER e SRTM mostraram resultados bastante semelhantes entre si, porém sua concordância com as curvas de nível originais das cartas do IBGE é muito baixa (Figura 5); apesar disto, ambos não removeram significativamente os picos presentes nas curvas de nível originais e neles também são verificadas curvas de nível que se cruzam e a ausência de uma curva de nível próxima da drenagem.

Para completar a avaliação qualitativa dos MDEs, conforme proposto por Wise (2000), foram derivadas as bacias de contribuição de cada MDE. A bacia do rio São Domingos digitalizada, foi sobreposta para ilustrar as principais diferenças entre esta e a bacia correspondente,
A.

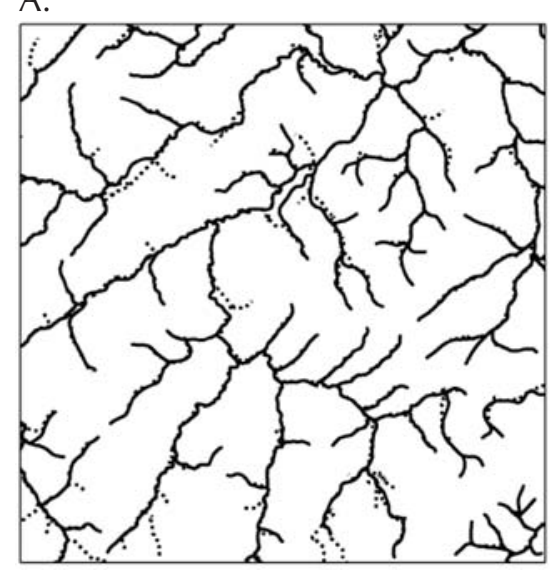

B.

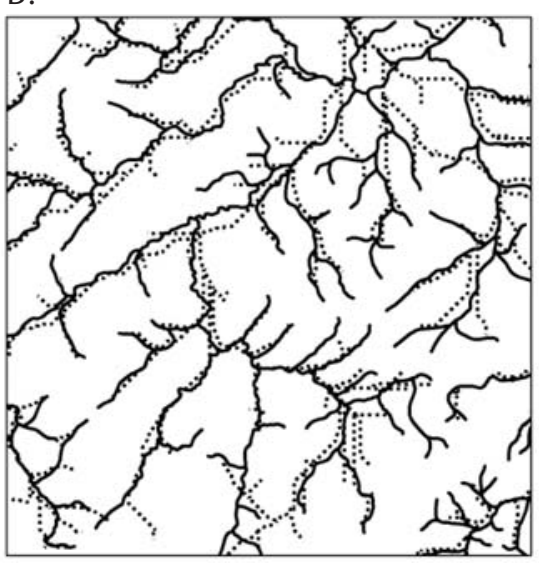

C.

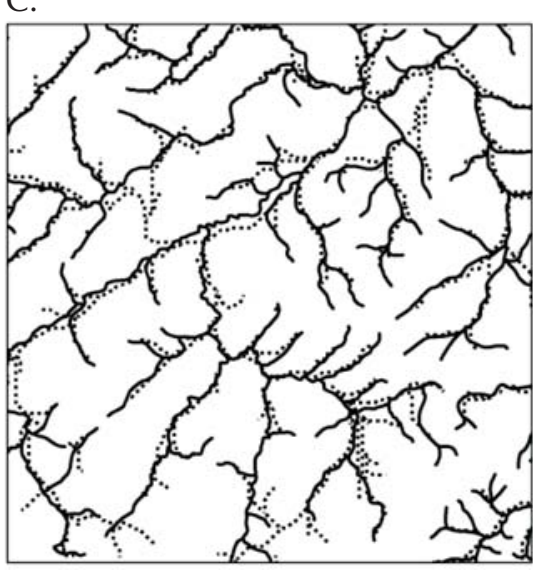

Figura 4. Comparação entre as redes de drenagem derivadas (linha tracejada) e a drenagem mapeada (linha cheia), em parte dos MDEs avaliados. A - MDE CARTA; B - MDE ASTER e C - MDE SRTM 
A.

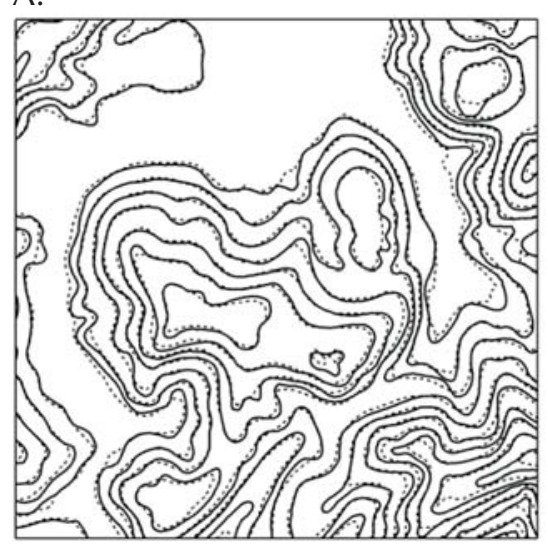

B.

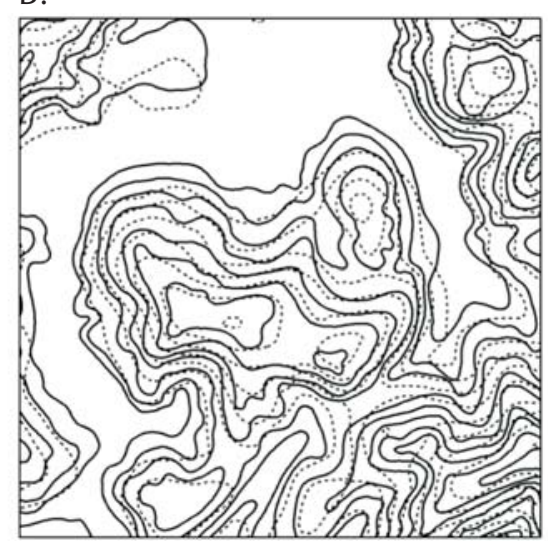

C.

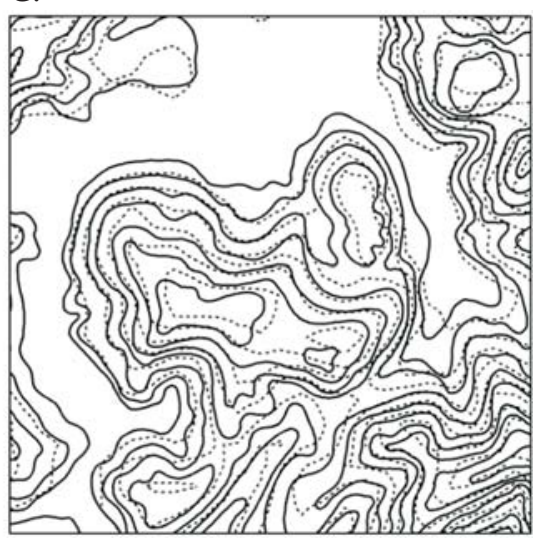

Figura 5. Curvas de nível derivadas (linha tracejada) versus curvas de nível originais (linha cheia), em parte dos MDEs avaliados. A - MDE CARTA; B - MDE ASTER e C - MDE SRTM

A.

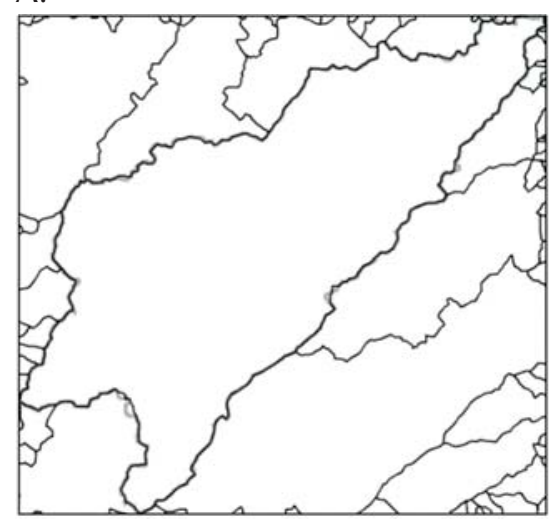

B.

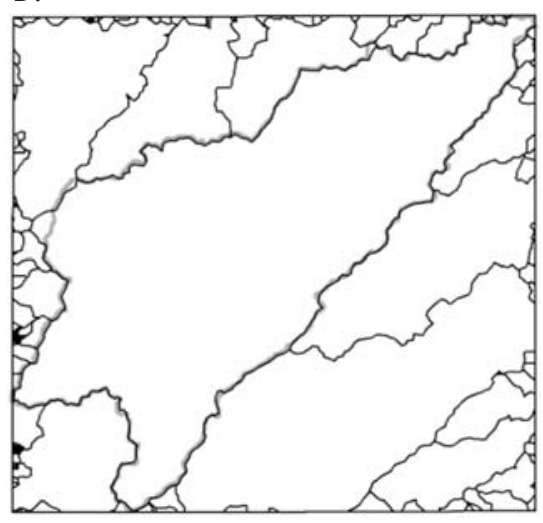

C.

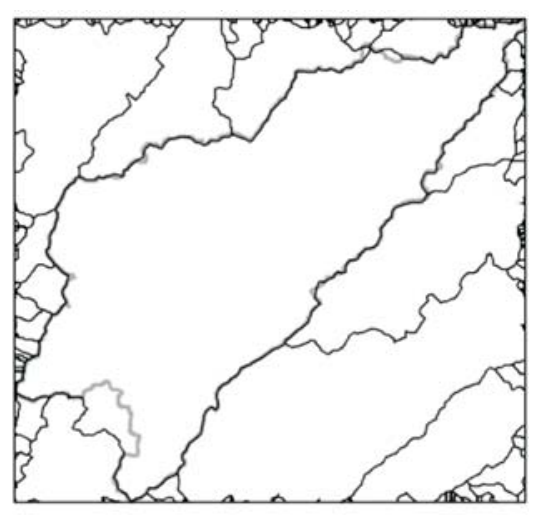

Figura 6. Bacias de contribuição derivadas dos MDEs. A - MDE CARTA; B - MDE ASTER e C - MDE SRTM

derivada pelos diferentes MDEs as quais, por sua vez, foram derivadas apenas pela identificação da direção do vizinho, com valor mais baixo de cada pixel e, desta maneira, são sensíveis à presença de artefatos nos dados. Pequenas diferenças na elevação entre pixels vizinhos podem, aparentemente, causar grandes mudanças na direção do fluxo e afetar, em conseqüencia, as bacias derivadas (Wise, 1998).

De maneira geral, verifica-se boa concordância entre os MDEs avaliados com relação às bacias de contribuição derivadas (Figura 6).

Os melhores resultados para esta avaliação foram obtidos para o MDE CARTA, que mostra boa concordância com o limite digitalizado da bacia do rio São Domingos. O limite da bacia derivada do MDE ASTER também mostrou boa concordância com a digitalizada já que, nas avaliações anteriores, este sempre apresentou os maiores problemas dentre os MDEs estudados; da mesma forma, o MDE SRTM, apresentou resultados bastante satisfatórios e ligeiramente inferiores aos MDEs CARTA e ASTER.

\section{CONCLUSÕES}

1. A análise qualitativa pelo RMSE mostrou que o MDE CARTA é superior aos demais, e que o RMSE não deve ser utilizado isoladamente para estimar a qualidade de MDEs.

2. Os resultados obtidos pelo MDE ASTER não foram satisfatórios, apresentando valores de RMSE significativamente mais elevados que os obtidos em outros estudos.

3. O MDE SRTM apresentou resultado quantitativo (RMSE) semelhante ao MDE CARTA e significativamente melhor do que o MDE ASTER.

4. O MDE CARTA apresentou, no geral, os melhores resultados e o mesmo pode ser utilizado para derivar atributos topográficos primários e secundários empregados no mapeamento digital de solos.

\section{LITERATURA CITADA}

Barros, R. S.; Cruz, C. B. M.; Reis, R. B.; Costa Júnior, N. A. Avaliação do modelo digital de elevação do SRTM na ortorretificação de imagens Landsat, área de aplicação: Angra dos Reis, RJ. In: Simpósio Brasileiro de Sensoriamento Remoto (SBSR), 12, 2005, Goiânia. Anais... São José dos Campos: INPE, 2005. p.3997-4004. CD-Rom

Bolch, T.; Kamp, U. Quality analyses of digital ASTER elevation models of high mountain areas (Cerro Sillajhuay, Chile/Bolivia). Kartographische Nachrichten, v.5, p.224-230, 2003.

R. Bras. Eng. Agríc. Ambiental, v.14, n.2, p.218-226, 2010. 
Bolstad, P. V.; Stowe, T. An evaluation of DEM accuracy - elevation, slope, and aspect. Photogrammetric Engineering and Remote Sensing, v.60, p.1327-1332, 1994.

Crepani, E.; Medeiros, J. S. Imagens fotográficas derivadas de MNT do projeto SRTM para fotointerpretação na geologia, geomorfologia e pedologia. São José dos Campos: INPE, 2004. 40p.

Cuartero, A.; Felicísimo, A. M.; Ariza, F. J. Accuracy of DEM generation from Terra-Aster stereo data. International Archives of Photogrammetry and Remote Sensing, v.35, p.225-260, 2004.

Dixon, T. H. SAR Interferometry and surface change detection. Miami: University of Miami, Rosenstiel School of Marine and Atmospheric Sciences, RASMAS TR 95-003, 1995. 97p.

Druzina, A. G. S.; Silva, J. L. B.; Zouain, R. N. A.; Souza, S. F. Estudo do erro de modelos digitais de elevação gerados a partir de imagens ASTER utilizando técnicas de processamento digital de imagens. Congresso Brasileiro de Cartografia, Simpósio de Geotecnologias para Petróleo e Exposicarta, 21, 2005, Macaé. Anais... Macaé: Sociedade Brasileira de Cartografia, Geodésia, Fotogrametria e Sensoriamento Remoto, 2005. CD-Rom.

Eckert, S.; Kellenberger, T. Quality analysis of automatic generated DEMs from ASTER-Data. Publikationen der Deutschen Gesellschaft für Photogrammetrie und Fernerkundung, v.11, p.337-345, 2002.

Falorni, G.; Teles, V.; Vivoni, E. R.; Bras, R. L.; Amaratunga, K. $\mathrm{S}$. Analysis and characterization of the vertical accuracy of digital elevation models from the Shuttle Radar Topography Mission. Journal of Geophysical Research - Earth Surface, v.110, p.F02005, 2005.

Felicísimo, A. M. Modelos digitales del terreno: Introducción aplicaciones en las ciencias ambientales. Oviedo: Universidad de Oviedo, 1994. <http://www.etsimo.uniovi.es/ feli>. 07 Nov. 2005.

Gerstenecker, C.; Läufer, G.; Steineck, D.; Tiede, C.; Wrobel, B. Validation of digital elevation models around Merapi Volcano, Java, Indonesia. Natural Hazards and Earth System Sciences, v.5, p.863-876, 2005.

Hengl, T.; Gruber, S.; Shrestha, D. P. Reduction of errors in digital terrain parameters used in soil-landscape modelling. International Journal of Applied Earth Observation and Geoinformation, v.5, p.97-112, 2004.

Hirano, A.; Welch, R.; Lang, H. Mapping from ASTER stereo image data: DEM validation and accuracy assessment. ISPRS Journal of Photogrammetry \& Remote Sensing, v.57, p.356-370, 2003.

Holmes, K.W.; Chadwick, O. A.; Kyriakidis, P. C. Error in a USGS 30-meter digital elevation model and its impact on terrain modeling. Journal of Hydrology, v.233, p.154-173, 2000.

Hutchinson, M. F.; Gallant, J. C. Digital elevation models and representation of terrain shape. In: Wilson, J. P.; Gallant, J. C. (ed.). Terrain analysis: Principles and applications. New York: John Wiley \& Sons, 2000. p.29-50.
Jarvis, A.; Rubiano, J.; Nelson, A.; Farrow, A.; Mulligan, M. Practical use of SRTM data in the tropics: comparisons with digital elevation models generated from cartographic data. Cali: Centro Internacional de Agricultura Tropical (CIAT), 2004. 32p. Working document n.198.

Kääb, A.; Huggel, C.; Paul, F.; Wessels, R.; Raup, B.; Kieffer, H.; Kargel, J. Glacier monitoring from ASTER imagery: accuracy and applications. In: EARSeL-LISSIG - Workshop Observing our Cryosphere from Space, 2, 2002, Bern. Proceedings... Bern: EARSeL, 2002. p.43-53.

Kamp, U.; Bolch, T.; Olsenholler, J. DEM generation from ASTER satellite data for geomorphometric analysis of Cerro Sillajhuay, Chile/Bolivia. In: Annual Meeting Imaging and Geospatial Information Society (ASPRS), 2003, Anchorage. Proceedings... Anchorage: ASPRS, 2003. CD-Rom

Kocak, G.; Büyüksalih, G.; Jacobsen, K. Analysis of digital elevation models determined by high resolution space images. International Archives of Photogrammetry and Remote Sensing, v.35, p.636-641, 2004.

Koch, A.; Heipke, C.; Lohmann, P. Analysis of SRTM DTM methodology and practical results. International Archives of Photogrammetry and Remote Sensing, v.34, p.470-475, 2002.

Poli, D.; Remondino, F.; Dolci, C. Use of satellite imagery for DEM extraction, landscape modeling and GIS applications. In: International Workshop on Processing and Visualization Using High-Resolution Imagery, 2004, Pitsanulok. Proceedings... Pitsanulok: ISPRS, 2004. CD-Rom

Ribeiro, C. A. S. Apostila de curso ENF613. Viçosa: UFV, 2003. 22p.

Santos, P. R. A.; Gaboardi, C.; Oliveira, L. C. Avaliação da precisão vertical dos modelos SRTM em diferentes escalas: um estudo de caso na Amazônia. In: Congresso Brasileiro de Cartografia, Simpósio de Geotecnologias para Petróleo e XXI Exposicarta, 22., 2005, Macaé. Anais... Macaé: Sociedade Brasileira de Cartografia, Geodésia, Fotogrametria e Sensoriamento Remoto, 2005. CD-Rom.

Subramanian, K. S. S.; Singh, A.; Sudhakar, M. Evaluation of digital elevation models created from different satellite images. 2005. <http://www.gisdevelopment.net/technology/rs/ mi03079pf.htm>. 12 Nov. 2005.

USGS - United States Geological Survey. Shuttle Radar Topography Mission (SRTM). 2005. <http://edc.usgs.gov/products/elevation/srtm>. 15 Nov. 2005.

Wise, S. The effect of GIS interpolation errors on the use of digital elevation models in geomorphology. In: Lane, S. N.; Richards, K. S.; Chandler, J. H. (ed.) Landform monitoring, modelling and Analysis. New York: John Wiley and Sons, 1998. 300p.

Wise, S. Assessing the quality for hydrological applications of digital elevation models derived from contours. Hydrological Processes, v.14, p.1909-1929, 2000. 